\title{
Riscos ergonômicos aos quais a equipe de Enfermagem está exposta em suas práticas
}

\section{laborais}

\author{
Ergonomic risks to which the Nursing team is exposed in their work practices \\ Riesgos ergonómicos a los que está expuesto el equipo de Enfermería en sus prácticas laborales
}

Recebido: 24/02/2021 | Revisado: 03/03/2021 | Aceito: 05/03/2021 | Publicado: 14/03/2021

Anakelle Oliveira dos Santos

ORCID: https://orcid.org/0000-0001-6526-2421

Faculdade Venda Nova do Imigrante, Brasil

E-mail: santosanakelle@gmail.com

Ilana Maria Brasil do Espírito Santo

ORCID: https://orcid.org/0000-0001-9591-2704

Centro Universitário Internacional UNINTER, Brasil

E-mail: ilaleao@outlook.com

Hallyson Leno Lucas da Silva

ORCID: https://orcid.org/0000-0001-7237-1706

Faculdades Integradas de Patos, Brasil

E-mail: hallysonlenolucas@hotmail.com

Alexsandra Maria Ferreira de Araújo Bezerra

ORCID: https://orcid.org/0000-0002-9306-581X

Sociedade Brasileira de Terapia Intensiva, Brasil

E-mail: alexsandrabezerra@ rocketmail.com

Joaffson Felipe Costa dos Santos

ORCID: https://orcid.org/0000-0001-5430-4518

Centro Universitário de Ciências e Tecnologia do Maranhão, Brasil

E-mail: fjjoaffson@outlook.com

Emília Vieira de Holanda Lira

ORCID: https://orcid.org/0000-0003-1466-4433

Universidade Federal do Maranhão, Brasil

E-mail: emilialira30@hotmail.com

Selminha Barbosa Bernardes Senna

ORCID: https://orcid.org/0000-0001-8359-3179

Universidade Federal do Piauí, Brasil

E-mail: selminhabernardes@ hotmail.com

Michelinne Shirley Pinheiro dos Santos

ORCID: https://orcid.org/0000-0002-1334-5345

Universidade Estadual do Piauí, Brasil

E-mail: michelinnesantos@yahoo.com.br

Francisca de Aquino Vieira Costa

ORCID: https://orcid.org/0000-0002-0260-9720

Faculdade Maurício de Nassau, Brasil

E-mail: gilkegil@hotmail.com

Alanna Nayra Araújo Azevêdo

ORCID: https://orcid.org/0000-0002-3901-4376

Faculdade Evangélica do Meio Norte, Brasil

E-mail: alannanayra144@gmail.com

Aline Stefhane Coutinho Coelho

ORCID: https://orcid.org/0000-0003-0933-4659

Centro Universitário UNINOVAFAPI, Brasil

E-mail: allynnesthephany@ hotmail.com

Camila Fernandes da Silva

ORCID: https://orcid.org/0000-0002-4712-6538

Centro Universitário Santo Agostinho, Brasil

E-mail: enfamila79@gmail.com

Iraci de Sousa e Silva

ORCID: https://orcid.org/0000-0003-4039-1504

CHRISFAPI, Brasil

E-mail: iracissilva@bol.com.br

Deylane de Melo Barros

ORCID: https://orcid.org/0000-0002-4803-4694

Centro Universitário UNINOVAFAPI, Brasil

E-mail: lanemelob@gmail.com 
Francisco Lucas de Lima Fontes

ORCID: https://orcid.org/0000-0003-1880-9329

Universidade Federal do Piauí, Brasil

E-mail: lucasfontesenf@ufpi.edu.br

\begin{abstract}
Resumo
Objetivou-se com o presente estudo analisar os riscos ergonômicos aos quais a equipe de Enfermagem se expõe em suas práticas laborais. Trata-se de uma revisão narrativa da literatura. As fontes para busca dos referenciais envolveram a base de dados Portal de Periódicos da Coordenação de Aperfeiçoamento de Pessoal de Nível Superior (CAPES), as bibliotecas Scientific Electronic Library Online (SciELO) e Biblioteca Virtual em Saúde (BVS) e o buscador eletrônico Google Scholar. A Enfermagem é uma das classes consideradas mais propícias a ocorrência de riscos ocupacionais, principalmente os ergonômicos. Os principais riscos ergonômicos observados para os profissionais dessa área são o levantamento de peso, a postura inadequada, bem como o esforço físico no trabalho. $\mathrm{O}$ empregado que também é exposto a intensas jornadas de trabalho sofre de desgaste físico que, no decorrer do tempo pode se converter em um agravo de saúde, ocasionando estresse, desmotivação, sonolência, queda da qualidade de trabalho e baixa autoestima. São necessárias a execução de medidas preventivas baseadas na ergonomia e na educação continuada como forma de proporcionar melhores condições de trabalho e qualidade de vida aos profissionais.
\end{abstract}

Palavras-chave: Saúde do trabalhador; Ergonomia; Enfermagem.

\begin{abstract}
The objective of this study was to analyze the ergonomic risks to which the Nursing team is exposed in their work practices. This is a narrative review of the literature. The sources for searching the references included the database of the Portal de Periódicos da Coordenação de Aperfeiçoamento de Pessoal de Nível Superior (CAPES), the libraries Scientific Electronic Library Online (SciELO) and the Biblioteca Virtual em Saúde (BVS) and the electronic search engine Google Scholar. The Nursing is one of the classes considered most conducive to the occurrence of occupational risks, especially ergonomic ones. The main ergonomic risks observed for professionals in this area are weight lifting, inadequate posture, as well as physical effort at work. The employee who is also exposed to intense working hours suffers from physical exhaustion that, over time, can become a health problem, causing stress, demotivation, drowsiness, drop in work quality and low self-esteem. Preventive measures based on ergonomics and continuing education are necessary to provide better working conditions and quality of life for professionals.
\end{abstract}

Keywords: Occupational health; Ergonomic; Nursing.

\title{
Resumen
}

El objetivo de este estudio fue analizar los riesgos ergonómicos a los que está expuesto el equipo de Enfermería en sus prácticas laborales. Esta es una revisión narrativa de la literatura. Las fuentes para la búsqueda de las referencias incluyeron la base de datos del Portal de Periódicos da Coordenação de Aperfeiçoamento de Pessoal de Nível Superior (CAPES), las bibliotecas Biblioteca Electrónica Científica en Línea (SciELO) y la Biblioteca Virtual em Saúde (BVS) y el buscador electrónico Google Scholar. Enfermería es una de las clases consideradas más propicias para la ocurrencia de riesgos laborales, especialmente los ergonómicos. Los principales riesgos ergonómicos observados para los profesionales de esta área son el levantamiento de pesas, la postura inadecuada, así como el esfuerzo físico en el trabajo. El empleado que además está expuesto a jornadas laborales intensas sufre un agotamiento físico que, con el tiempo, puede convertirse en un problema de salud, provocando estrés, desmotivación, somnolencia, caída en la calidad del trabajo y baja autoestima. Las medidas preventivas basadas en la ergonomía y la educación continua son necesarias para brindar mejores condiciones laborales y calidad de vida a los profesionales.

Palabras clave: Salud laboral; Ergonomía; Enfermería.

\section{Introdução}

O trabalho é imprescindível ao homem, possui relevante utilidade na autorrealização, colabora com a construção identitária do trabalhador, possibilita retorno financeiro e conquistas aos propósitos da vida. Todavia, no instante em que o sujeito se insere no mundo laboral fica sujeito a riscos ocupacionais que, hoje, são considerados um importante problema de saúde pública por repercutir nas condições de vida dos trabalhadores. Dentre os riscos aos quais os empregados estão expostos merece destaque o ergonômico. Tal risco pode ser definido como qualquer evento que ocasione disfunções psicológicas e fisiológicas ao empregado, gerando a este ator sérios problemas de saúde (Andrade, Santos \& Torres, 2018; Neves et al., 2018; Silva et al., 2021).

Com o intento de compreensão de termos como risco, saúde, doença e trabalho entra, nesse contexto, a saúde 
ocupacional, que relaciona-se à segurança e à qualidade de vida dos trabalhadores. A partir da detecção de fatores que interfiram na saúde do empregado, são delimitadas ações de prevenção, rastreamento e diagnóstico de agravos relacionados a doenças laborais. Os profissionais da saúde encontram-se expostos aos mesmos riscos que os demais trabalhadores, acrescidos à exposição a materiais biológicos (sangue e fluidos contaminados) (Souza et al., 2017; Andrade et al., 2020; Marques et al., 2020).

Dentre os profissionais da saúde que merecem atenção, destacam-se os profissionais da Enfermagem, os quais possuem diversas funções manuais e sociais a partir do contato direto com pacientes. A atuação desses trabalhadores fundamenta-se no cuidado direto com o indivíduo e indireto com a busca de melhorias para complementar o embasamento técnico-prático da saúde. Em geral, a classe da Enfermagem atua sob elevadas jornadas de trabalho, acúmulo de funções e em ambientes insalubres, que a deixa vulnerável a inúmeros riscos ocupacionais causados por fatores químicos, mecânicos, físicos, ergonômicos e biológicos, incluindo os psicossociais (Loro, Bittencourt \& Zeitoune, 2017; Arcanjo et al., 2018;). Com o intuito de garantir melhores condições aos trabalhadores, o Ministério da Saúde tem buscado adotar políticas preventivas à saúde do trabalhador, definindo estratégias para o desenvolvimento de atenção integral aos profissionais (Brasil, 2018).

Por meio da ergonomia é possível colaborar para mudanças nas condições e no ambiente laboral, mediante adaptações nos recursos e no processo de trabalho para melhorar a qualidade de vida dos empregados. A ergonomia também volta-se à prevenção dos danos causados aos trabalhadores durante o desempenho de suas funções, de modo a reduzir riscos, sem prejuízo ao desempenho profissional. Assim, os riscos ergonômicos refletem diretamente na produtividade, uma vez que causam danos físicos e mentais nos profissionais. Devido suas inúmeras consequências é essencial o mapeamento dos riscos ergonômicos em unidades de saúde, de modo a identificar os setores com maior exposição. A ergonomia busca o planejamento e práticas que favoreçam a saúde do trabalhador, a fim de que estes atores desempenhem suas atividades sem que coloquem sua saúde em risco (Nascimento et al., 2019; Silva et al., 2019).

Dessa forma, a Norma Regulamentadora 17 (NR-17), que trata da ergonomia no ambiente de trabalho, surgiu com o objetivo de definir critérios que oportunizem a adaptação das condições de trabalho aos aspectos psicofisiológicos dos empregados, possibilitando máximo conforto, segurança e desempenho eficiente do empregado em suas práticas laborais (Brasil, 1990). Ficam claros os variados fatores envolvidos no manejo de um ambiente de trabalho salutar, como postura, condições ambientais, organização, conforto, disponibilização de equipamentos, entre outros. Diante do brevemente explanado, este estudo teve como objetivo analisar os riscos ergonômicos aos quais a equipe de Enfermagem se expõe em suas práticas laborais.

\section{Metodologia}

Trata-se de uma revisão narrativa da literatura, de caráter exploratório e descritivo com abordagem qualitativa. Dentre os tipos de revisões de literatura, a narrativa é considerada a mais tradicional. Também chamada de bibliográfica, é uma revisão ampla, com temas discutidos de maneira global, sem preocupação em esgotar as fontes de informações. Nela, exploram-se referenciais diversos e não há exigência de procedimentos rígidos para sua construção (Cordeiro et al., 2007). A opção pela abordagem qualitativa deste estudo ocorreu porque esse tipo de pesquisa lida, preferencialmente, com dados descritivos. A pesquisa qualitativa fornece o significado, as percepções e as descrições que os sujeitos dão aos fenômenos. Nela, o processo analítico dos dados e informações segue, comumente, o processo indutivo (Pereira et al., 2018).

As fontes para busca dos referenciais envolveram a base de dados Portal de Periódicos da Coordenação de Aperfeiçoamento de Pessoal de Nível Superior (CAPES), as bibliotecas Scientific Electronic Library Online (SciELO) e Biblioteca Virtual em Saúde (BVS) e o buscador eletrônico Google Scholar.

Por tratar-se de uma revisão narrativa a procura por materiais em mais de uma fonte ocorreu para ampliar o leque de 
caminhos para o desenvolvimento do estudo. Na busca dos materiais adotaram-se os seguintes Descritores em Ciências da Saúde (DeCS): "saúde do trabalhador", "ergonomia" e "Enfermagem". Foram incluídos materiais (artigos científicos, monografias, dissertações, teses e demais produtos técnicos sobre a temática estudada) disponíveis na íntegra e em línguas portuguesa, inglesa ou espanhola. Optou-se por não aplicar recorte temporal na busca dos materiais.

Os materiais foram selecionados de modo casual, buscando-se produções que respondessem ao objetivo estabelecido pela pesquisa e temática estudada. Logo, as compreensões que se realizaram dos materiais ficaram sujeitas ao viés de seleção, com interpretações e análise crítica subjetivo a cargo dos autores da revisão. Para discussão foram incluídos 23 referenciais.

\section{Resultados e Discussão}

Para a manutenção de um ambiente de trabalho saudável e produtivo é indispensável que este seja livre de riscos e que, sobretudo, garanta a segurança e a saúde dos funcionários que nele atuam. A Norma Regulamentadora 10 (NR-10) define o risco como a "capacidade de grandeza com potencial para causar lesões ou danos à saúde das pessoas" (Brasil, 1983).

O sistema de saúde encontra-se voltado para a prevenção e recuperação da saúde de seus pacientes. Contudo, ao atuarem nesse campo os profissionais passam a se expor a inúmeras atividades consideradas insalubres e/ou perigosas originárias de condições ou técnicas de trabalho (Macinko \& Harris, 2015). Para Carrara, Magalhães e Lima (2015), os riscos ocupacionais de tais atividades afetam diretamente a vida do empregado. Acredita-se que os profissionais da saúde, principalmente os enfermeiros, encontram-se constantemente expostos estes riscos, uma vez que os hospitais, um dos estabelecimentos de saúde que o enfermeiro pode atuar, são considerados insalubres, deixando os trabalhadores expostos a riscos químicos, biológicos, físicos psíquicos e mecânicos.

Segundo a Organização Internacional do Trabalho, ocorrem anualmente 2,34 milhões de mortes decorrentes de acidentes ou doenças ocasionados no trabalho. Destas, cerca de 6.300 mortes acontecem em seus ambientes de trabalho e 5.500 causadas por doenças decorrentes de suas práticas profissionais (OIT, 2013). Autores como Barbosa et al. (2020) consideram esses dados inaceitáveis, afirmando que ações necessitam ser implementadas nesse âmbito de modo a garantir segurança, equidade e condições adequadas de trabalho.

Os riscos ocupacionais são classificados em seis categorias: biológico, mecânico, psicossocial, ergonômico, de acidente de trajeto e físico. Os riscos biológicos são aqueles relacionados à exposição do trabalhador a agentes infecciosos, tais como bactérias, fungos, entre outros. Nos riscos químicos, incluem-se substâncias, produtos ou compostos que penetram no corpo do trabalhador. No campo dos riscos ergonômicos pode-se listar repetitividade, levantamento de peso, má postura e ritmo excessivo de trabalho. Os riscos de acidentes de trajeto incluem a eletricidade, a probabilidade de incêndio ou até mesmo casos de explosão. Os riscos físicos, incluem ruído, calor, frio, pressão, umidade, iluminação e vibração. Há ainda riscos ocupacionais oriundos de mudanças tecnológicas e sociais advindas da globalização, assim como psicossocial (Fonseca et al., 2015; Moss et al., 2016).

No Brasil, a ergonomia é normatizada pela NR-17, a qual foi criada a partir de uma reivindicação sindical para o reconhecimento de doenças atreladas ao trabalho (Bernal et al., 2015). Cavalcante \& Teles (2012) afirmam que a NR-17 visa favorecer a adaptação das condições laborais a características psicofisiológicas dos funcionários, incluindo informações atreladas à organização do trabalho, a condições ambientais, aos equipamentos, ao mobiliário e aos transportes.

Os riscos ergonômicos estão relacionados com diversos fatores e situaç̃es que podem afetar o trabalhador. Dentro da área da saúde, os profissionais de Enfermagem são considerados um dos grupos de maior risco ergonômico, podendo estar relacionados com a prática laborais diárias (Rocha et al., 2016; Souza, Cortez \& Carmo, 2017). Os principais riscos ergonômicos observados para os profissionais dessa área são o levantamento de peso, a postura inadequada, bem como o esforço físico no trabalho (Pasa et al., 2015). Com isso, nota-se o surgimento de dores no corpo e doenças osteomusculares 
como a Lesão por Esforço Repetitivo (LER) e Distúrbios Osteomusculares Relacionado ao Trabalho (DORT) (Paula; Sanchez; Pereira, 2018).

Os trabalhadores da Enfermagem possuem prevalência de 43 a 93\% no desenvolvimento de LER/DORT, sendo este um dos principais motivos pelos quais esses profissionais são afastados de suas atividades (Paula, Sanchez \& Pereira, 2016). A LER/DORT são consideradas afecções de músculos, tendões, nervos e ligamentos que muitas vezes levam o indivíduo a incapacidade temporária ou permanente. Relacionam-se ao surgimento de dor crônica e parestesia (Valença \& Alencar, 2015; Martins, Correia \& Vidal, 2017). A dor na região lombar também é uma das principais queixas dos profissionais da Enfermagem. Quando essa dor torna-se crônica é observado o comprometimento das atividades exercidas no trabalho, bem como na sua vida social (Freire, Soares \& Torres, 2017).

A remoção, movimentação e rolamento de pacientes realizados pela equipe de Enfermagem tem sido responsável pelo desenvolvimento de dor na região lombar, seja o ato realizado em ambiente hospitalar ou em circunstâncias variadas. O risco ergonômico relacionado com o levantamento de peso pelo profissional pode ter várias relações como a dependência total do paciente, a quantidade de aparelhos a que este está ligado, bem como a falta de equipamentos auxiliares para o transporte do enfermo (Leite et al., 2016; Oliveira, Pelissari \& Matoski, 2016).

É necessária detecção de quais riscos ergonômicos a equipe de Enfermagem está sujeita, com o propósito de realizar a prevenção de distúrbios osteomusculares, bem como a recuperação da saúde do profissional, quando for o caso. Para prevenir esses distúrbios devem ser utilizadas as abordagens ergonômicas, conhecendo os profissionais e as atividades desenvolvidas por eles, além de ouvir sugestões dos trabalhadores para melhores condições de trabalho (Carraca, Magalhães \& Lima, 2015; Oliveira, Pelissali \& Matoski, 2016).

As condições laborais ofertadas aos profissionais também merecem destaque, especialmente as associadas ao turno de trabalho, quando foca-se em plantões noturnos. O empregado que é exposto a intensas jornadas de trabalho sofre de desgaste físico que, no decorrer do tempo pode se converter em um agravo de saúde, ocasionando estresse, desmotivação, sonolência, queda da qualidade de trabalho e baixa autoestima (Souza, 2003). No estudo de Santana et al. (2019) constatou-se que enfermeiro, em especial, apresentam nível significativo de estresse e as situações que mais causam este agravo associam-se à relação com outros setores e supervisores. Ademais, os relacionamentos interpessoais também podem ser causadores de estresse ocupacional entre os profissionais.

Para prevenir o desenvolvimento de lesões é essencial que os profissionais tenham conhecimentos sobre os riscos ergonômicos a que estão suscetíveis. Com isso, os enfermeiros do trabalho devem promover atividades com teor educativo e preventivo, incentivando os profissionais a novos hábitos, reeducação postural, bem como a realização de pausas durante o dia, respeitando seu tempo de descanso (Marques et al., 2015). Algumas medidas utilizadas para minimizar esses riscos podem ser a ginastica laboral e a musicoterapia, que podem proporcionar alívio de estresse, bem como relaxamento desses trabalhadores (Paula, Sanchez \& Pereira, 2018; Santos, Silva \& Passos, 2016).

\section{Considerações Finais}

O entendimento dos princípios ergonômicos é bastante relevante na compreensão do comportamento humano para as atividades laborais e da vida diária. A procura por cenários seguros e saudáveis dentro do ambiente de trabalho visa a proteção e preservação da vida humana e, sobretudo, é um modo de garantir melhor qualidade de vida aos trabalhadores. Os profissionais de Enfermagem expõem-se a diversos riscos ergonômicos: esforço físico e levantamento de peso no manejo dos pacientes acamados, postura inadequada durante a realização de procedimentos, exigência de alta produtividade, situações estressantes, plantões noturnos, intensas jornadas de trabalho, esforços repetitivos, entre outros.

São necessárias a execução de medidas preventivas baseadas na ergonomia e na educação continuada como forma de 
proporcionar melhores condições de trabalho e qualidade de vida aos profissionais. Nesse contexto, o enfermeiro com ênfase em saúde do trabalhador pode ser o profissional mais indicado para, juntamente com o estabelecimento de saúde, tomar medidas para minimizar os riscos ergonômicos e promover a saúde da equipe de Enfermagem. Assim, além de ofertar mais qualidade de vida aos profissionais, também melhora a produtividade da instituição e a diminuição das taxas de absenteísmo.

Desse modo, a compreensão sobre os riscos ergonômicos aponta para a necessidade de maiores estudos empíricos que discutam a temática, com aprofundamento dos desafios e das perceptivas que a equipe de Enfermagem possui quanto a sua capacidade produtiva, qualidade de vida laboral e autocuidado nas práticas profissionais.

\section{Referências}

Andrade, B. B., Santos, L. F., \& Torres, L. M. (2018) Os riscos ergonômicos no cotidiano das equipes de Enfermagem. Revista Relações Sociais, 1(3), 04980510 .

Andrade, G. B., Siqueira, H. C. H., Yasin, J. C. M., Soares, L. S., Medeiros, A. C., \& Silva, D. N. (2020). Ações de prevenção dos riscos à saúde e qualidade de vida do enfermeiro e usuário. Research, Society and Development, 9(4), e72943043.

Arcanjo, R. V. G., Christovam, B. P., Souza, N. V. D. O., Silvino, Z. R., \& da Costa, T. F. (2018). Conocimientos y prácticas de los trabajadores de enfermería sobre riesgos laborales en la atención primaria de salud: un estudio de intervención. Enfermería Global, 17(3), $200-237$.

Barbosa, B. S. T., Ferreira, L. G. S., Lima, J. C., Oliveira, R. M. M., Souza, L.A., Walsh, I. A. P. (2020). Síndrome de burnout, variáveis sociodemográficas, ocupacionais e satisfação no trabalho na equipe de Enfermagem hospitalar. Revista Família, Ciclos de Vida e Saúde no Contexto Social, 8(2), 232-241.

Bernal, D., Serna, J. C., Tobias, A., Prada, S. V., Benavides, F. G., Serra C. (2015). Work-related psychosocial risk factors and musculoskeletal disorders in hospital nurses and nursing aides: a systematic review and meta-analysis. International Journal of Nursing Studies, 52(2), 635-648.

Brasil. (2018). Ministério da Saúde. Secretaria de Atenção à Saúde. Secretaria de Vigilância em Saúde. Saúde do trabalhador e da trabalhadora. Brasília: Ministério da Saúde.

Brasil. (1983). Ministério do Trabalho e da Previdência Social. Norma Regulamentadora 10 (NR-10). Brasília: Ministério do Trabalho e da Previdência Social. Brasil. (1990). Ministério do Trabalho e da Previdência Social. Norma Regulamentadora 17 (NR-17). Brasília: Ministério do Trabalho e da Previdência Social.

Carrara, G. L. R., Magalhães, D. M., \& Lima, R. C. (2015). Riscos ocupacionais e os agravos à saúde dos profissionais de enfermagem. Revista Fafibe Online, 8(1), 265-286.

Cavalcante, I. C. J. B, \& Teles, N. S. B. (2012). Riscos ergonômicos aos quais os profissionais de enfermagem estão expostos em ambiente hospitalar: uma revisão da literatura. Revista Diálogos Acadêmicos, 1(1), 39-48.

Cordeiro, A. M., Oliveira, G. M., Rentería, J. M., \& Guimarães, C. A. (2007). Revisão sistemática: uma revisão narrativa. Revista do Colégio Brasileiro de Cirurgiões, 34(6), 428-431.

Dias, C., Damasceno, J., Silva, L., \& Rocha, B. (2020). Saúde do profissional de Enfermagem: riscos ocupacionais em ambiente hospitalar. Saúde, 46(2), e34972.

Fonseca, F. F., Costa, F. M., Lima, C. A., Silva, S. S. S., Alves, J. P., \& Carneiro, J. A. (2016). Caracterização do risco ocupacional entre trabalhadores da Estratégia Saúde da Família. Revista Brasileira de Pesquisa em Saúde, 17(2), 89-97.

Freire, L. A., Soares, T. C. N., \& Torres, V. P. S. (2017). Influência da ergonomia na biomecânica de profissionais de Enfermagem no ambiente hospitalar. Biológicas \& Saúde, 7(24), 72-80.

Leite, H. C. M., Cariman, S. A. E., Silva, N., \& Carvalho, A. (2017). Risco ocupacional entre profissionais de saúde do serviço de atendimento móvel de urgência - SAMU. Enfermagem em Foco, 7(3/4), 31-35.

Loro, M. M., Bittencourt, V. L. B., \& Zeitoune, R. C. G. (2017). Pesquisa convergente assistencial: equipe de Enfermagem compartilhando saberes sobre riscos ocupacionais e propondo intervenções. Revista Mineira de Enfermagem, 21, e-1044.

Macinko, J., \& Harris, M. J. (2015). Brazil's family health strategy--delivering community-based primary care in a universal health system. The New England Journal of medicine, 4,372(23), 2177-2181.

Marques, D. O., Pereira, M. S., Souza, A. C. S., Vila, V. S. C., Almeida, C. C. O. F., \& Oliveira, E. C. (2015). O absenteísmo - doença da equipe de enfermagem de um hospital universitário. Revista Brasileira de Enfermagem, 68(5), 876-882.

Marques, Y. L. V., Barros, D. M., Santos, M. C. M. C., Balica, N. M. P., Santo, I. M. B. E., Bezerra, A. M. F. A. (2020). Exposição ocupacional por material biológico nas práticas assistenciais de Enfermagem. International Journal of Development Research, 10(11), 42470-42474.

Martins, E. A., Correa, C. S., \& Vidal, P. C. V. (2017). Doenças osteomusculares relacionadas ao trabalho com destaque aos profissionais da área de Enfermagem. Revista Perspectiva: Ciência e Saúde, 2(2), 107-118. 
Research, Society and Development, v. 10, n. 3, e24610313259, 2021

(CC BY 4.0) | ISSN 2525-3409 | DOI: http://dx.doi.org/10.33448/rsd-v10i3.13259

Moss, M., Good, V. S., Gozal, D., Kleinpell, R., Sessler, C. N. (2016) An Official Critical Care Societies Collaborative Statement: Burnout Syndrome in Critical Care Healthcare Professionals: A Call for Action. Critical Care Medicine, 44(7), 1414-21.

Nascimento, T. R. L., Siqueira, J. C. F., Gonçalves, S. B., Silva, N. L., \& Souza, E. L. (2019). Ergonomia: saúde ocupacional e qualidade de vida. Revista Ação Ergonômica, 13(1), 151-162.

Neves, D. R., Nascimento, R. P., Felix Júnior, M. S., Silva, F. A., \& Andrade, R. O. B. (2018). Sentido e significado do trabalho: uma análise dos artigos publicados em periódicos associados à Scientific Periodicals Electronic Library. Cadernos EBAPE.BR, 16(2), 318-330.

Oliveira, J. M. C., Pelissari, V., \& Pelissari, A. (2015). Movimentação e transporte de pacientes - riscos ergonômicos. Revista Engenharia \& Construção Civil, 2(1), 19-28.

Organização Internacional do Trabalho (OIT). (2013). La prevención de las enfermedades profesionales. Genebra: OIT.

Pasa, T. S., Magnago S. T. S. B., Silva, R. M., Cervo, A. S., Beck, C. L. C., \& Viero, N. C. (2015). Riscos ergonômicos para trabalhadores de Enfermagem ao movimentar e remover pacientes. Revista de Enfermagem da UFSM, 5(1), 92-102.

Paula, A. A., Sanchez, M. C. O., \& Pereira, M. J. (2018). Lesões por Esforço Repetitivo/Distúrbio Osteomuscular Relacionado ao Trabalho: contribuições para a prevenção em trabalhadores de enfermagem. ACC CIETNA: Revista de la Escuela de Enfermería, 3(2), 6-18.

Pereira, A. S., Shitsuka, D. M., Parreira, F. J., Shitsuka, R. (2018). Metodologia da Pesquisa Científica. $1^{a}$ ed. Santa Maria: UFSM, NTE.

Rocha, R. O., Vieira, N. S., Santos, L. D., Pena, M. S. P., Madureira, T. R. (2016). A importância da ergonomia no trabalho do profissional de enfermagem relacionado ao cuidado com o paciente totalmente dependente. Revista de Trabalhos Acadêmicos, 2(1), 1-36.

Santana, R. S., Fontes, F. L. L., Morais, M. J. A., Costa, G. S., Silva, R. K., Araújo, C. S., Silva, A. L. B., Pereira, R. I. N. (2019). Occupational stress among emergency and urgent care nurses at a public hospital in Teresina, Piaui, Brazil. Revista Brasileira de Medicina do Trabalho, 17(1), 76-82.

Santos, E., Silva, R., \& Passos, V. (2016). Contribuição do enfermeiro do trabalho na prevenção primária relacionado a riscos ergonômicos. Revista Uningá Review, 26(1), 68-73.

Silva, M. L., Silva, M. P. B., Leite, A. C., Oliveira, E. F. P., Fernandes, A. R. C. A., Jatobá, D. N. V. (2021). A ergonomia no ambiente de trabalho dos enfermeiros do SAMU: uma visão da Enfermagem. Research, Society and Development, 10(1), e30410111552.

Silva, V., Gracini, R., Melo, J. C., Tavares, P. H., Geribello, R., \& Amarante, M. (2019). A correlação entre ergonomia e saúde ocupacional. Revista Pesquisa e Ação, 5(1), 109-126.

Souza, G. F., Ferreira, A. P., Moreira, M. F. R., Portela, L. F. (2017). Fatores de riscos ocupacionais e implicações à saúde do trabalhador em biotérios. Saúde em Debate, 41(S2), 188-199.

Souza, S. R. R. K. (2003). A rotatividade e as condições de trabalho dos profissionais de Enfermagem: um enfoque da ergonomia. Dissertação (mestrado). Universidade Federal de Santa Catarina (UFSC), Florianópolis.

Souza, V., Cortez, E. A., \& Carmo, T. G. (2017). Medidas educativas para minimizar os riscos ocupacionais na equipe de enfermagem da UTI. Revista de Pesquisa: Cuidado é Fundamental Online, 9(2), 583-591.

Valença, J. B. M., \& Alencar, M. C. B. (2015). Distúrbios osteomusculares e o trabalho de técnicos e auxiliares de Enfermagem em instituições de idosos. $O$ Mundo da Saúde, 39(3), 316-324. 